\title{
Joint Learning of Laser Relative Intensity and Frequency Noise from Single Experiment
} and Single Detected Quadrature

\author{
Brajato, Giovanni; Zibar, Darko
}

Published in:

Proceedings of European Conference on Optical Communication

Link to article, DOI:

10.1109/ECOC.2018.8535269

Publication date:

2018

Document Version

Peer reviewed version

Link back to DTU Orbit

Citation (APA):

Brajato, G., \& Zibar, D. (2018). Joint Learning of Laser Relative Intensity and Frequency Noise from Single Experiment and Single Detected Quadrature. In Proceedings of European Conference on Optical Communication IEEE. https://doi.org/10.1109/ECOC.2018.8535269

\section{General rights}

Copyright and moral rights for the publications made accessible in the public portal are retained by the authors and/or other copyright owners and it is a condition of accessing publications that users recognise and abide by the legal requirements associated with these rights.

- Users may download and print one copy of any publication from the public portal for the purpose of private study or research.

- You may not further distribute the material or use it for any profit-making activity or commercial gain

- You may freely distribute the URL identifying the publication in the public portal 


\title{
Joint Learning of Laser Relative Intensity and Frequency Noise from Single Experiment and Single Detected Quadrature
}

\author{
G. Brajato(1) and D. Zibar ${ }^{(1)}$
}

(1) DTU Fotonik, Technical University of Denmark, Build. 343, DK-2800, Denmark dazi@fotonik.dtu.dk

\begin{abstract}
Bayesian inference framework, that considers laser-physics, is proposed and demonstrated for joint learning of laser static and dynamic parameters. Proof-of-concept experimental results demonstrating the main concepts are presented as well.
\end{abstract}

\section{Introduction}

Accurate characterization of lasers sources in terms of relative intensity noise (RIN) and frequency noise (FN) power spectrum density (PSD) is of great significance for the design of highspeed short-range and long-haul optical links ${ }^{1}$. Even though laser RIN and FN PSD characterization is an integral part of laser science, there are still many unsolved challenges remaining, such as $^{2}:$ 1) measurement noise originating from electrical and optical amplifiers, receivers front-ends and sampling scopes, 2) two separate measurements are typically needed, 3 ) both quadratures of the detected laser field are needed and 4 ) various non-white FN components $\left(1 / f^{\alpha}, \alpha \neq 0\right)$ may hide the true laser intrinsic linewidth.

The core of the laser RIN and FN characterization is the estimation of the photon number (power) and optical phase. Various methods for laser RIN and FN characterization have been proposed $^{1-3}$ and references therein. However, the majority of the proposed methods do not offer an optimum and joint estimation of photon number and optical phase in the presence of the measurement noise. We have recently demonstrated that the techniques from machine learning can be used to perform optimum optical phase detection and thereby enable accurate laser FN characterization ${ }^{4,5}$. The presented framework used Bayesian state-space based inference and employed small signal laser rate equation. Most importantly, the laser static parameters were computed offline and required some heuristics.

In this paper, a Bayesian inference framework for joint learning of laser static and dynamic parameters (carrier and photon number, and optical phase) is proposed and numerically demonstrated by employing Unscented Kalman Filtering (UKF) and Metropolis Hasting (MH) sampling algorithm. The framework takes large-signal laser rate equations into account and as well as laser frequency drift. The main principles behind the joint learning of static and dynamic parameters are evaluated on the experimental data using a single quadrature of the detected laser field, simplifying the measurement procedure significantly.

\section{Theoretical framework}

A numerical simulation is employed first to test the proposed framework. The laser is simulated by coupled rate equations driven by Langevin noise sources ${ }^{6}$. The rate equations describe the relation between carrier number, $N(t)$, photon number, $S(t)$, and optical phase, $\phi(t)$. These are considered to be laser dynamic parameters. The normalized output of the laser is thereby expressed as: $y(t)=\sqrt{S(t)} e^{i\left(\omega_{0} t+D t^{2}+\phi(t)\right)}+n(t)$, where $\omega_{0}$ is the optical angular frequency, $D$ is laser linear frequency drift $\left[\mathrm{Hz}^{2} / \mathrm{Hz}\right]$ and $n(t)$ is measurement noise with Gaussian distribution. The objective is estimation of the photon number $S(t)$ and the optical phase $\phi(t)$ from $y(t)$ by employing UKF$\mathrm{MH}$ algorithm. It is assumed that the coherent detection is employed to detect the laser output field followed by the Bayesian inference framework. For the method employed, it is assumed that the local oscillator (LO) has negligible intensity and frequency noise compared to the laser under the test. The state-space model at the receiver is expressed as following:

$$
\begin{array}{r}
{\left[\begin{array}{c}
N_{k} \\
S_{k} \\
\phi_{k}
\end{array}\right]=\mathbf{F}\left(\left[\begin{array}{c}
N_{k-1} \\
S_{k-1} \\
\phi_{k-1}
\end{array}\right], \boldsymbol{\Theta}\right)+\boldsymbol{\Gamma}_{k-1}} \\
{\left[\begin{array}{c}
y_{k}^{i} \\
y_{k}^{q}
\end{array}\right]=} \\
{\left[\begin{array}{c}
\sqrt{S_{k}} \cos \left(\Delta \omega m+D m^{2}+\phi_{k}\right) \\
\sqrt{S_{k}} \sin \left(\Delta \omega m+D m^{2}+\phi_{k}\right)
\end{array}\right]+\left[\begin{array}{c}
n_{k}^{i} \\
n_{k}^{q}
\end{array}\right]}
\end{array}
$$

where $m=k T_{s}, k$ is an integer, $T_{s}$ is the sampling time, $\mathbf{F}(\cdot)$ represents the deterministic 

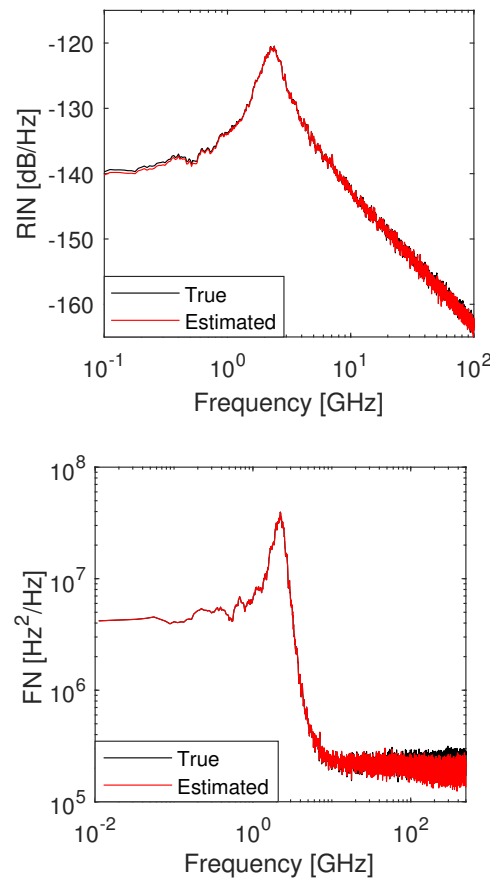

Fig. 1: Comparison of the true (black) and estimated (red) RIN and FN power spectrum density using the model in equation (1).

part of rate equations, $\boldsymbol{\Gamma}(\cdot)$ represents Langevin noise forces, $\Delta \omega$ is the angular frequency offset between the laser under the test and the LO, and finally $\Theta$ represents laser static parameters such as: carrier lifetime $\tau_{n}$, photon lifetime $\tau_{p}$, gain slope $g$, nonlinear gain compression factor $\epsilon$, carrier number at transparency $N_{0}$, fraction of spontaneous emission $\beta$, linewidth enhancement factor $\alpha$ and the time-average carrier number $\bar{N}$. The objective is to estimate laser dynamic parameters $S_{k}$ and $\phi_{k}$ from the received measurements $y_{l}^{i}$ and $y_{k}^{q}$. However, the laser static parameters $\Theta$ are not known and therefor they must be jointly estimated with the dynamic parameters. Joint static and dynamic parameter learning is achieved by embedding Metropolis-Hastings algorithm within Unscented Kalman Filtering framework. A general description of the framework is presented in ${ }^{7}$. The framework is fully Bayesian as the posterior probability of the static and dynamic parameters is obtained which is unique for the method, allowing for the measurement uncertainty quantification. Finally, the framework is general meaning that it can include any form of rate-equations including the multi-modal and temperature effects.

\section{Numerical results}

In this section, the state-space model from equation (1) is applied for joint learning of laser static and dynamic parameters. It is assumed that the initial values of the static laser parameters are within $\pm 20 \%$ of the true laser parameters, even though, initial values within $\pm 40 \%$ are actually tolerable with a slight decrease in the performance. In Fig. 1, the true and the estimated RIN and FN PSD are shown. It is observed that the true and the estimated curves overlap fully, demonstrating the feasibility of the accurate and joint RIN and FN PSD estimation from a single time-series of the detected data.

The advantage of the state-space model in equation (1) is that it is general. However, the disadvantage is that it includes a relatively large number of unknown laser static parameters. Next, a simpler and more generic state-space model, equation (2), is proposed that can also capture the laser dynamics correctly.

$$
\begin{array}{r}
{\left[\begin{array}{c}
S_{k}^{\prime} \\
\phi_{k}^{\prime}
\end{array}\right]=\left[\begin{array}{l}
S_{k-1}^{\prime} \\
\phi_{k-1}^{\prime}
\end{array}\right]+\left[\begin{array}{c}
\Gamma_{k-1}^{S^{\prime}} \\
\Gamma_{k-1}^{\phi^{\prime}}
\end{array}\right]} \\
{\left[\begin{array}{c}
y_{k}^{i} \\
y_{k}^{q}
\end{array}\right]=} \\
{\left[\begin{array}{c}
S_{k}^{\prime} \cos \left(\Delta \omega m+D m^{2}+\phi_{k}^{\prime}\right) \\
S_{k}^{\prime} \sin \left(\Delta \omega m+D m^{2}+\phi_{k}^{\prime}\right)
\end{array}\right]+\left[\begin{array}{c}
n_{k}^{i} \\
n_{k}^{q}
\end{array}\right]}
\end{array}
$$

The advantage of the simplified state-space model in equation (2) is that there are less static parameters to be estimated compared to the complete model in (1). The unknown static parameters are the variances associated with the noise sources of the squared root photon number, $S_{k}^{\prime}$ and the optical phase, $\phi_{k}^{\prime}$, i.e. $\left[\Gamma_{k}^{S^{\prime}}, \Gamma_{k}^{\phi^{\prime}}\right]$ and the measurement noise variance. Coarse estimate of the offset frequency $\Delta \omega$ and laser linear frequency drift $D$ can be obtained by taking $\angle\left(y_{k}^{i}+i y_{k}^{q}\right)$ and employing a quadratic fit. These estimate can later be improved by the $\mathrm{MH}$ sampling algorithm.

The estimated RIN and FN power spectrum density using the simplified state-space model from equation (2) are shown in Fig. 2. It is observed that the true and the estimated RIN and FN PSD fully overlap. This is a very interesting result as the model describing the squared photon number and optical phase is specified as a Wiener process which has white FN PSD. However, the estimated RIN and FN PSD are nonwhite. and follow the true ones. This is because in the update step of the UKF, the measurements $\left[y_{k}^{i}, y_{k}^{q}\right]$ play more importance than the predictions governed by the Wiener process. This is valid as long as the measurement noise is not too large. 

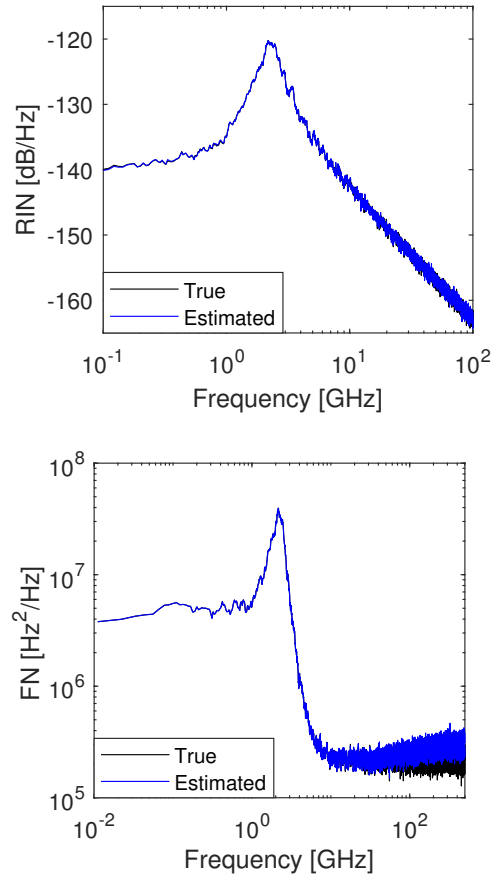

Fig. 2: Comparison of the true (black) and estimated (blue) RIN and FN power spectrum density using the model in equation (2).

\section{Experimental results}

Next, the state-space model from equation (2) in combination with the Bayesian inference employing the UKF and the MH sampling algorithm is tested on the experimental data. The proposed framework only requires a single quadrature component of the detected optical field, i.e. $y_{k}^{i}$, The estimated RIN and FN PSD are shown in Fig. 3.

For comparisons, RIN and FN PSD obtained by conventional approaches ${ }^{1-3}$, that all need both quadratures components, $y_{k}^{i}$ and $y_{k}^{q}$, and do not employ optimum detection, are also shown. Fig. 3 demonstrates that by employing the UKF-MH algorithm, it is feasible to perform joint and accurate photon number and optical phase estimation from a single detected quadrature. The proposed method (UKF-MH), results in a significantly lower FN PSD compared to the conventional approach, due to the measurement noise filtering and removal of the $1 / f^{\alpha}$ component. Finally, for the RIN PSD, the proposed method reveals some relevant features such as resonance peaking as well as noise filtering at high frequencies.

\section{Conclusion and perspectives}

It has been showed numerically and experimentally that the Bayesian inference framework using the unscented Kalman filtering combined with Metropolis-Hastings algorithm can be used to jointly and accurately estimate laser RIN and FN power spectrum density. Finally, it has been
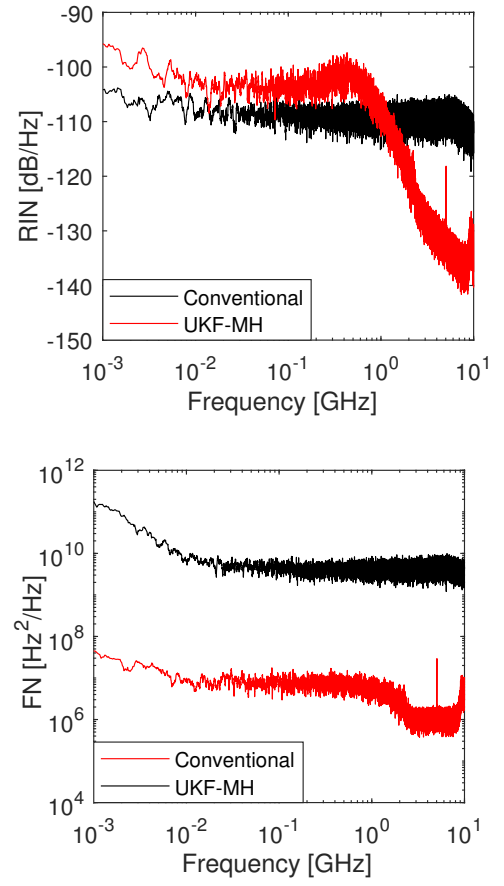

Fig. 3: Experiemntal data: Estimated RIN and FN PSD employing the state-space model in equation (2) and the UKF-MH algorithm.

demonstrated that the optical phase estimation is feasible using a single detected quadrature of the optical field. This can potentially have significant benefits for the carrier recovery for continues variable quantum key distribution (CV-QKD) systems, where typically only a single quadrature is detected, as the phase diversity detection, $[\sin (\cdot)$, $\cos (\cdot)]$, results in a $3 \mathrm{~dB}$ penalty.

\section{References}

[1] N. von Bandel et al., "Time-dependent laser linewidth: beat-note digital acquisition and numerical analysis," Optics Express, vol. 24, (24), (2016)

[2] M. Schiemangk et al., "Accurate frequency noise measurement of free-running lasers," Applied Optics, vol. 53, no. 20, (2014)

[3] K. Kikuchi "Characterization of semiconductor laser phase noise and estimation of bit-error rate performance with low-speed offline digital coherent receivers," Optics Express, vol. 20, (5), 2012

[4] M. Piels et al., "Laser rate equation-based filtering for carrier recovery in characterization and communication," $\mathrm{J}$. Lightwave Technol. vol. 33, no. 15, (2016)

[5] D. Zibar et al., "Machine learning under the spotlight," Nature Photonics, 11, (2017)

[6] L. Coldren et al., "Diode lasers and photonic circuits," John Wiley and Sons, (2012)

[7] J. Dahlin and T. B. Schon, "Getting started with particle Metropolis-Hastings for inference in nonlinear dynamical models," Journal of Statistical Software (JSS), (2018) 